\title{
A Thermoregulatory Behavior
}

\author{
Andrew C. Gallup \\ Department of Biological Sciences, Binghamton University, Binghamton, N.Y., USA
}

\begin{abstract}
The existence of yawning across vertebrate species suggests important basic functions, and the spontaneous and involuntary nature of a yawn lends support for it having adaptive significance. Recent research suggests the biological function of yawning among homeotherms is central thermoregulation. Comparative research from birds, rats and humans shows that yawning reduces brain and body temperature, is influenced by the range and direction of ambient temperature change, and is inhibited by methods of behavioral cooling. This research provides strong support for the view that yawning stimulates or facilitates cortical arousal, demonstrating that a yawn is a behavioral response to transient brain hyperthermia, acting to counter intermittent increases in brain temperature and promote thermal homeostasis. This theory is powerful because it not only integrates a great deal of seemingly diverse information about yawning, but it can also be used to generate numerous testable predictions. Applications from this research range from basic physiological understanding to the improved treatment and understanding of diseases associated with thermoregulatory dysfunction.

Copyright ๑ 2010 S. Karger AG, Basel
\end{abstract}

Yawning is characterized by a large gaping of the mouth and eye closure, accompanied by a deep inhalation of air and a shorter expiration. Phylogenetically old, yawning has been documented in all 5 classes of vertebrates, suggesting it has important basic functions [1]. Although the mere occurrence of yawning across species is not evidence that it is an evolved adaptation, the spontaneous and involuntary nature of yawning lends support for its adaptive significance. Likewise, similar to other adaptive responses such as sneezing, yawning provides a brief sense of gratification in healthy populations [2]. To this point, however, attempts to identify the functional and primitive significance of yawning have led to little consensus [3].

One of the more widespread hypotheses attempting to explain the physiological purpose of yawning posits a role in respiration. In particular, a commonly held view is that yawning functions to equilibrate oxygen levels in the blood; however, this hypothesis remains unsupported. Provine et al. [4] showed that while breathing heightened levels of oxygen or carbon dioxide increased breathing rates, it did 
not influence yawning [4]. They also demonstrated that physical exercise sufficient to double breathing rates has no effect on yawning. Therefore, contrary to popular belief, it has been concluded that yawning does not serve a respiratory function, and that yawning and breathing are controlled by separate mechanisms.

Baenninger [5] has reviewed a vast amount of literature that suggests yawning functions to stimulate or facilitate arousal during state change. In line with this hypothesis, yawning occurs in anticipation of important events and during behavioral transitions or changes in activity levels across vertebrate taxa. Accordingly, it has been proposed that the adaptive function of yawning is to modify levels of cortical arousal. However, while this account may explain the hedonic quality and unprompted nature of yawning, the particular physiological mechanism for achieving such arousal has not been identified. Here, this chapter will describe the most recent empirical evidence indicating a role of yawning in central thermoregulation.

\section{'Brain Cooling Hypothesis'}

Brain temperature is determined by a number of variables, including the temperature of the blood supplying the brain, rate of blood flow through the brain, and rate of metabolic heat production. Cooling mechanisms include increased circulation, vasodilation, convection and conduction processes, heat dissipation through the upper airways, and the presence of venous plexuses or intertwined venous and arterial rete structures [6]. Recent research suggests/supports that yawning is involved in central thermoregulation [7-9]. According to this hypothesis, spontaneous yawning is triggered in response to mild central hyperthermia, acting to counter intermittent increases in brain temperature and promote thermal homeostasis. Thus, it is proposed that a yawn increases arousal and mental efficiency by maintaining optimal brain temperature.

It is well known that most instances of yawning tend to occur shortly after awakening and before sleeping $[10,11]$. Body temperature begins to peak in the evening and the onset of sleep initiates a decline in core body temperature. Likewise, the tendency to wake-up occurs in tandem with a rise in core body temperature in the morning. Therefore consistent with the brain cooling hypothesis, yawning frequently occurs in the evening, when brain temperature is at its peak, and upon waking, when brain temperature begins increasing from its lowest point [12]. Yawning in early morning may be a compensatory thermal stabilizing mechanism. Waking disrupts a relaxed state, and rapid increases in movement and conscious brain activity produce ensuing metabolic increases in temperature. By lowering brain temperature and maintaining thermal homeostasis, this theory suggests that instead of prompting sleep, yawning actually serves to maintain focus and attention, thereby antagonizing sleep [7]. Furthermore, prolonged sleep deprivation in rats has been shown to increase deep brain temperature [13]. Subjective ratings of sleepiness in humans correlate with 
increases in skin temperature while lying down [14], and with increases in core body temperature when standing [15]. In addition, hot water consumption has been shown to increase body temperature as well as sleepiness, while ice intake produces the converse [16]. Therefore, it appears that variations in body temperature are associated with corresponding variations in sleepiness, and this may explain why people often yawn when they are tired [11].

Pharmacological research has demonstrated that yawning is under the control of the hypothalamus [17], and structures of the hypothalamus have been strongly linked to thermoregulation [18]. In support of the brain cooling hypothesis, the physiological consequences of a yawn are in accord with those that would be necessary for cerebral cooling $[7,8]$. For instance, the deep inhalation of air taken into the lungs causes an acceleration in heart rate $[19,20]$ and also raises blood pressure [21]. More specifically, the constriction and relaxation of facial muscles during a yawn increases facial blood flow, and these changes are thought to increase cerebral blood flow [22, 23]. Together these processes may act like a radiator by removing hyperthermic blood from specific areas while introducing cooler blood from the lungs and extremities, thereby cooling cortical surfaces through convection. It is also hypothesized that the gaping of the mouth and deep inhalation during a yawn cools venous blood draining from the nasal and oral orifices into the cavernous sinus, which surrounds the internal carotid artery supplying blood to the rest of the brain. Thus, the temperature of the air is proposed to give a yawn its utility, not the air's composition.

\section{Empirical Support for the 'Brain Cooling Hypothesis'}

Initial support for the brain cooling hypothesis came from a report showing that nasal breathing and direct forehead cooling diminished the incidence of yawning in humans [7]. Given that previous research has shown nasal breathing and forehead cooling each reduce brain temperature $[6,24]$, it was suggested that the need for yawning may have been eliminated when the brain was sufficiently cooled by nasal cavity airflow or arterial blood supply. Recent research has shown that brain surface temperature can be lowered by mechanical introduction of cool air into the nasal cavity [25], and further evidence using intracranial thermocouples demonstrates nasal inhalation can reduce frontal lobe brain temperature by as much as $0.8^{\circ} \mathrm{C}$ [24]. Likewise, forehead cooling reduces the temperature of the blood in cutaneous and subcutaneous venous plexuses, and this cooled blood can be transported through venous communications into the venous plexuses of the dura matter [6]. Forehead cooling also enhances dissipation of heat from the skull, and the cooling of venous blood by the skin can in turn alter arterial (carotid) blood supply to the brain.

In order to directly test the brain cooling hypothesis, more recent research has now investigated actual changes in brain and body temperature before and after yawning. In a case report of two women suffering from chronic bouts of yawning, one woman 
recorded her oral temperature before and after yawning episodes. She reported the onset of a yawning episode occurred during mild hyperthermia $\left(37.5^{\circ} \mathrm{C}\right)$, and then resulted in an average decrease in body temperature of roughly $0.4^{\circ} \mathrm{C}$ following each yawning attack [26]. In addition, both women reported that nasal breathing and forehead cooling (i.e. behavioral brain cooling methods) provided symptom relief and/ or postponement of yawning attacks. Thus, empirical evidence reveals that excessive yawning reduces human body temperature. To more explicitly investigate the potential brain cooling effects of yawning, Shoup et al. [27] took continuous measures of brain temperature before and after yawning by implanting rats with thermocoupled temperature probes in the frontal cortex. Consistent with the predictions of the brain cooling hypothesis, spontaneous yawning was triggered in response to rapid increases in brain temperature $\left(+0.12^{\circ} \mathrm{C}\right)$, followed by corresponding decreases in temperature immediately following a yawn. These results confirm the hypothesis that yawning produces cerebral cooling. While $\pm 0.12^{\circ} \mathrm{C}$ might seem trivial, this amounts to roughly $25 \%$ of the circadian variance in brain temperature among humans [12].

\section{Further Predictions and Support of the Brain Cooling Model}

This evidence suggests the mechanism that triggers yawning lies within a hierarchy of neural structures controlling temperature regulation, offering numerous testable predictions. For one, it is expected that yawning should occur in a narrow range of ambient temperatures (i.e. a thermal window) [7, 8]. Yawns should increase in frequency as ambient temperature approaches body temperature, but should cease when ambient temperatures reach or exceed body temperature because they would no longer result in cooling. It is assumed that during the rise in ambient temperature, the body is heating, stimulating thermoregulatory mechanisms to control temperatures within a normal range. Likewise, when temperatures fall below a certain point, yawning would no longer be beneficial because sending a wave of unusually cool blood to the brain would be maladaptive.

The first prediction was recently tested using budgerigars (Melopsittacus undulatus) as an avian model [9]. Results showed that budgerigars' yawning rates were significantly influenced by increasing ambient temperatures, providing the first documented evidence that yawning may be a temperature regulating mechanism in birds. More specifically, yawning frequency increased during rising ambient temperature, but when temperature approached body temperature, yawning began to diminish as other evaporative heat loss behaviors became prevalent. In order to distinguish between whether yawning was triggered due to exposure to higher or increasing ambient temperature, or simply by change in temperature (irrespective of direction), a more recent study manipulated ambient temperature in both directions and confirmed that yawning occurred significantly more during high-increasing temperatures compared to decreasing temperatures [28]. In addition, yawning was positively 
correlated with ambient temperature and occurred significantly more often in the presence of other thermoregulatory behaviors.

Existing research suggests that excessive yawning is not always indicative of sleep deprivation or sleep disorder, and may be a more important symptom of an individual's inability to properly maintain thermal homeostasis [29]. Gallup and Gallup [8] have reviewed mounting evidence showing that yawning occurs before, during and after instances of heat stress, hyperthermia and abnormal thermoregulation. For instance, drugs that increase brain temperature produce excessive yawning (e.g. certain serotonin reuptake inhibitors), while drugs that produce hypothermia (e.g. opioids) inhibit yawning. Excessive yawning is also symptomatic of conditions that increase brain and/ or core body temperature, such as central nervous system damage, epilepsy, migraine headaches, multiple sclerosis, and stress and anxiety. It is hypothesized that frequent yawning is a symptom of an inability to effectively maintain optimal brain thermoregulation, and therefore yawning may be indicative of hyperthermia. Recent research on patients with multiple sclerosis, a documented thermoregulatory disorder, shows that over 1 out of 3 individuals indicate that yawning provides temporary relief of their MS symptoms [30]. Similarly, recent pilot data show that some epileptic patients notice yawning before and after seizures and $50 \%$ of these patients reported that yawning made their symptoms slightly better [Gallup AC, Gallup Jr. GG, unpubl. data]. It is proposed that the alleviatory effects described by such patients are a result of cerebral cooling.

\section{Conclusions}

Overall, this research provides strong support for the view that yawning stimulates or facilitates cortical arousal, with growing evidence suggesting the biological function of yawning among homeotherms is central thermoregulation. Comparative research from birds, rats and humans shows that yawning reduces brain and body temperature, is influenced by the range and direction of ambient temperature change, and is inhibited by methods of behavioral cooling (e.g. nasal breathing and forehead cooling). Further support comes from the fact that excessive yawning is frequently associated with conditions of heat stress, hyperthermia and abnormal thermoregulation, and drugs and other conditions that promote increases in brain and/or body temperature have been shown to increase yawning frequency. This model is powerful because it not only integrates a great deal of seemingly diverse information about yawning from a variety of species, but it can also be used to generate numerous testable predictions.

The evidence presented in this chapter cannot be overlooked or ignored, as it offers direct application for medical research and diagnosis, as well as providing critical insights to sleep medicine. Yawning has well-documented thermoregulatory consequences, and the use of thermocoupled probes in rats confirms the hypothesis that yawning provides cerebral cooling. The fact of the matter is that the 'brain cooling hypothesis' is no longer a topic of debate. 


\section{References}

1 Baenninger R: Some comparative aspects of yawning in Betta splendens, Homo sapiens, Panthera leo, and Papio sphinx. J Comp Psychol 1987;101:349354.

-2 Provine RR: Yawning as a stereotyped action pattern and releasing stimulus. Ethology 1986;72:109122.

$>3$ Provine RR: Yawning. Am Sci 2005;93:532-539.

$\checkmark 4$ Provine RR, Tate BC, Geldmacher LL: Yawning: no effect of $3-5 \% \mathrm{CO}_{2}, 100 \% \mathrm{O}_{2}$, and exercise. Behav Neural Biol 1987;48:382-393.

5 Baenninger R: On yawning and its functions. Psychonomic Bull Rev 1997;4:198-207.

-6 Zenker W, Kubik S: Brain cooling in humans - anatomical considerations. Anat Embryol (Berl) 1996; 193:1-13

7 Gallup AC, Gallup GG Jr: Yawning as a brain cooling mechanism: nasal breathing and forehead cooling diminish the incidence of contagious yawning. Evol Psychol 2007;5:92-101.

$>8$ Gallup AC, Gallup Jr GG: Yawning and thermoregulation. Physiol Behav 2008;95:10-16.

$>9$ Gallup AC, Miller ML, Clark AB: Yawning and thermoregulation in budgerigars (Melopsittacus undulatus). Anim Behav 2009;77:109-113.

10 Provine RR, Hamernik HB, Curchack BB: Yawning: relation to sleeping and stretching in humans. Ethology 1986;76:152-160.

$>11$ Zilli I, Giganti F, Salzarulo P: Yawning in morning and evening types. Physiol Behav 2007;91:218-222.

$\checkmark 12$ Landolt HP, Moser S, Wiesen HG, Borbeley AA, Dijk DJ: Intracranial temperature across 24 hour sleep wake cycles in humans. Neuroreport 1995; 6:913-917.

13 Everson CA, Smith CB, Sokoloff L: Effects of prolonged sleep deprivation on local rates of cerebral energy metabolism in freely moving rats. J Neurosci 1994;14:6769-6778.

14 Krauchi K, Cajochen C, Wirz-Justice A: A relationship between heat loss and sleepiness: effects of postural change and melatonin administration. J Appl Physiol 1997;83:134-139.

15 Krauchi K, Cajochen C, Wirz-Justice A: Thermophysiological aspects of the three-process-model of sleepiness regulation. Clin J Sport Med 2005;24:287300 .

\section{A.C. Gallup}

Department of Biological Sciences, Binghamton University

Binghamton, NY-13902 USA

Tel. +1 518727 2473, Fax +1 6077776521

E-Mail a.c.gallup@gmail.com
16 Krauchi K, Vollenweider S, Muller S, Renz C, Schachinger H, Wirz-Justice A: Thermoregulatory dissociation of awareness and psychomotor vigilance (abstract). Sleep 2006;29(suppl):A34.

17 Argiolas A, Melis MR: The neuropharmacology of yawning. Eur J Pharmacol 1998;343:1-16.

18 Cooper KE: Molecular biology of thermoregulation: some historical perspectives on thermoregulation. J Appl Physiol 2002;92:1717-1724.

19 Greco M, Baenninger R: Effects of yawning and related activities on skin conductance and heart rate. Physiol Behav 1991;50:1067-1069.

20 Guggisberg AG, Mathis J, Herrmann US, Hess CW: The functional relationship between yawning and vigilance. Behav Brain Res 2007;179:159-166.

21 Askenasy JJM, Askenasy N: Inhibition of muscle sympathetic nerve activity during yawning. Clin Auton Res 1996;6:237-239.

22 Askenasy JJM: Is yawning an arousal defense reflex? J Psychol 1989;123:609-621.

23 Zajonc RB: Emotion and facial efference: a theory reclaimed. Science 1985;288:15-21.

24 Mariak Z, White MD, Lewko J, Lyson T, Piekarski P: Direct cooling of the human brain by heat loss from the upper respiratory tract. J Appl Physiol 1999;87: 1609-1613.

25 Harris BA, Andrews PJD, Murray GD: Enhanced upper respiratory tract airflow and head fanning reduce brain temperature in brain-injured, mechanically ventilated patients: a randomized, crossover, factorial trial. Br J Anesth 2007;98:93-99.

26 Gallup GG Jr, Gallup AC: Yawning and thermoregulation: two case histories of chronic, debilitating yawning. Sleep Breath, E-pub ahead of print.

27 Shoup ML, Gallup AC, Gallup GG Jr, McNay EC: Yawning cools the brain. Under review.

28 Gallup AC, Miller ML, Clark AB: The direction and range of ambient temperature influences yawning in budgerigars (Melopsittacus undulatus). J Comp Psychol, in press.

29 Gallup AC: Misconceptions regarding excessive yawning and sleep deprivation; in Fulke P, Vaughan S (eds): Sleep Deprivation: Causes, Effects, and Treatment. Hauppauge, Nova Science, in press.

30 Gallup AC, Gallup GG Jr, Feo C: Yawning, sleep, and symptom relief in patients with multiple sclerosis. Sleep Med, in press. 